DOI: $10.2478 /$ awutm-2013-0010<smiles>CC1=CC=C1</smiles>

VERSITA
Analele Universităţii de Vest,

Timişoara

Seria Matematică - Informatică

LI, 1, (2013), 153- 166

\title{
On Sandwich Theorems for Analytic Functions Defined by a Certain Generalized Linear Operator
}

Tamer Mohamed Seoudy

\begin{abstract}
We obtain some applications of first order differential subordination and superordination results involving certain linear operator and other linear operators for certain normalized analytic functions. Some of our results improve and generalize previously known results.
\end{abstract}

AMS Subject Classification (2000). Primary: 26A33, 30C80. Secondary: 30C45, 33C15.

Keywords. Analytic function, Hadamard product, differential subordination, differential superordination, linear operator, sandwich results

\section{Introduction}

Let $H(U)$ be the class of analytic functions in the open unit disk $U=\{z \in$ $\mathbb{C}:|z|<1\}$ and let $H[a, k]$ be the subclass of $H(U)$ consisting of functions of the form

$$
f(z)=a+a_{k} z^{k}+a_{k+1} z^{k+1} \ldots(a \in \mathbb{C}) .
$$


For simplicity we denote $H[a]=H[a, 1]$. Also, let $\mathcal{A}$ be the subclass of $H(U)$ consisting of functions of the form

$$
f(z)=z+\sum_{k=2}^{\infty} a_{k} z^{k} .
$$

If $f, g \in H(U)$, we say that $f$ is subordinate to $g$ or $f$ is superordinate to $g$, written $f(z) \prec g(z)$ if there exists a Schwarz function $\omega$, which (by definition) is analytic in $U$ with $\omega(0)=0$ and $|\omega(z)|<1$ for all $z \in U$, such that $f(z)=g(\omega(z)), z \in U$. Furthermore, if the function $g$ is univalent in $U$, then we have the following equivalence, (cf., e.g., [6], [16] and [17]):

$$
f(z) \prec g(z) \Leftrightarrow f(0)=g(0) \text { and } f(U) \subset g(U) .
$$

Let $\phi: \mathbb{C}^{2} \times U \rightarrow \mathbb{C}$ and $h$ be univalent in $U$. If $p$ is analytic in $U$ and satisfies the first order differential subordination

$$
\phi\left(p(z), z p^{\prime}(z) ; z\right) \prec h(z),
$$

then $p$ is a solution of the differential subordination (3). The univalent function $q$ is called a dominant of the solutions of the differential subordination (1.3) if $p \prec q$ for all $p$ satisfying (3). A univalent dominant $\tilde{q}$ that satisfies $\tilde{q} \prec q$ for all dominants of (3) is called the best dominant. If $p$ and $\phi\left(p(z), z p^{\prime}(z) ; z\right)$ are univalent in $U$ and if $p$ satisfies first order differential superordination

$$
h(z) \prec \phi\left(p(z), z p^{\prime}(z) ; z\right),
$$

then $p$ is a solution of the differential superordination (4). An analytic function $q$ is called a subordinant of the solutions of the differential superordination (4) if $q \prec p$ for all $p$ satisfying (4). A univalent subordinant $\tilde{q}$ that satisfies $q \prec \tilde{q}$ for all subordinants of (4) is called the best subordinant. Using the results of Miller and Mocanu [17], Bulboaca [5] considered certain classes of first order differential superordinations as well as superordinationpreserving integral operators [6]. Ali et al. [1], have used the results of Bulboaca [5] to obtain sufficient conditions for normalized analytic functions to satisfy the double subordination

$$
q_{1}(z) \prec \frac{z f^{\prime}(z)}{f(z)} \prec q_{2}(z),
$$

where $q_{1}$ and $q_{2}$ are given univalent functions in $U$ with $q_{1}(0)=q_{2}(0)=1$. Also, Tuneski [25] obtained a sufficient condition for starlikeness of $f$ in 
terms of the quantity $\frac{f^{\prime \prime}(z) f(z)}{\left(f^{\prime}(z)\right)^{2}}$. Recently, Shanmugam et al. [24] obtained sufficient conditions for the normalized analytic function $f$ to satisfy

$$
q_{1}(z) \prec \frac{f(z)}{z f^{\prime}(z)} \prec q_{2}(z)
$$

and

$$
q_{1}(z) \prec \frac{z^{2} f^{\prime}(z)}{\{f(z)\}^{2}} \prec q_{2}(z) .
$$

In [24] they obtained results for functions defined by using Carlson-Shaffer operator [7], Ruscheweyh derivative [20] and Sălăgean operator [22].

For the functions $f$ given by 1 and $g \in \mathcal{A}$ given by

$$
g(z)=z+\sum_{k=2}^{\infty} b_{k} z^{k}
$$

the Hadamard product (or convolution) of $f$ and $g$ is defined by

$$
(f * g)(z)=z+\sum_{k=2}^{\infty} a_{k} b_{k} z^{k}=(g * f)(z) .
$$

For the functions $f, g \in \mathcal{A}$, we define the linear operator $D_{\lambda}^{n}: \mathcal{A} \rightarrow \mathcal{A}$ $\left(\lambda \geq 0, l \geq 0 ; n \in \mathbb{N}_{0}=\mathbb{N} \cup\{0\}, \mathbb{N}=\{1,2, \ldots\}\right)$ by

$$
\begin{aligned}
D_{\lambda, l}^{n}(f * g)(z)= & D_{\lambda, l}\left(D_{\lambda, l}^{n-1}(f * g)(z)\right) \\
= & z+\sum_{k=2}^{\infty}\left[\frac{l+1+\lambda(k-1)}{l+1}\right]^{n} a_{k} b_{k} z^{k} \\
& \left(\lambda \geq 0 ; l \geq 0 ; n \in \mathbb{N}_{0}\right) .
\end{aligned}
$$

From (7) we can easily deduce that

$$
\lambda z\left(D_{\lambda, l}^{n}(f * g)(z)\right)^{\prime}=(l+1) D_{\lambda}^{n+1}(f * g)(z)-(l+1-\lambda) D_{\lambda, l}^{n}(f * g)(z) .
$$

We observe that the linear operator $D_{\lambda, l}^{n}$ reduces to several interesting many other linear operators considered earlier for different choices of $n, \lambda, l$ and the function $g$ :

(i) $D_{\lambda, 0}^{n}=D_{\lambda}^{n}$, where $D_{\lambda}^{n}$ is linear operator which was defined by Aouf and Mostafa [3];

(ii) For $g(z)=\frac{z}{1-z}$, we have $D_{\lambda, l}^{n}(f * g)=I(n, \lambda, l) f$, where $I(n, \lambda, l)$ is the generalized multiplier transformation which was introduced and studied by Cătaş et al. [8]; 
(iii) For $\lambda=1$ and $g(z)=\frac{z}{1-z}$, we see that $D_{1, l}^{n}(f * g)=I(n, l) f$, where $I(n, l) f(z)$ is the multiplier transformation (see [9]);

(iv) For $l=0$ and $g(z)=\frac{z}{1-z}$, we see that $D_{\lambda, 0}^{n}(f * g)=D_{\lambda}^{n}$ where $D_{\lambda}^{n}$ is the generalized Sălăgean operator [2] which yield Sălăgean operator $D^{n}$ for $\lambda=1$ introduced and studied by Sălăgean [22];

(v) For $l=0$ and

$$
\begin{gathered}
g(z)=z+\sum_{k=2}^{\infty} \Gamma_{k}\left[a_{1} ; b_{1}\right] z^{k} \\
\Gamma_{k}\left[a_{1} ; b_{1}\right]=\frac{\left(a_{1}\right)_{k-1} \ldots\left(a_{q}\right)_{k-1}}{\left(b_{1}\right)_{k-1} \ldots\left(b_{s}\right)_{k-1}(1)_{k-1}} \\
\left(a_{i} \in \mathbb{C} ; i=1, . ., q ; b_{j} \in \mathbb{C} \backslash \mathbb{Z}_{0}^{-}=\{0,-1,-2, . .\} ; j=1, . ., s ; q \leq s+1 ; q, s \in \mathbb{N}_{0}\right),
\end{gathered}
$$

where

$$
(x)_{k}= \begin{cases}1 & \text { if } k=0 ; x \in \mathbb{C}^{*}=\mathbb{C} \backslash\{0\} \\ x(x+1) \ldots(x+k-1) & \text { if } k \in \mathbb{N} ; x \in \mathbb{C}\end{cases}
$$

we have $D_{\lambda, 0}^{n}(f * g)=D_{\lambda}^{n}\left(a_{1}, b_{1}\right) f$, where $D_{\lambda}^{n}\left(a_{1}, b_{1}\right)$ is the linear operator which was introduced and studied by Selvaraj and Karthikeyan [23]. The operator $D_{\lambda}^{n}\left(a_{1}, b_{1}\right)$, contains in turn many interesting operators such as Dziok-Srivastava operator [10] ( see also [11]), Hohlov linear operator (see [13]), the Carlson-Shaffer linear operator (see [7] and [21] ), the Ruscheweyh derivative operator (see [20]), the Bernardi-Libera-Livingston operator ( see [4], [14] and [15]) and Owa-Srivastava fractional derivative operator (see [19]); (iv) For $g$ of the form (9) we obtain

$$
I_{q, s, \lambda}^{n, l}\left(a_{1}, b_{1}\right) f(z)=z+\sum_{k=2}^{\infty}\left[\frac{l+1+\lambda(k-1)}{l+1}\right]^{n} \Gamma_{k}\left[a_{1} ; b_{1}\right] z^{k}
$$

where the operator $I_{q, s, \lambda}^{n, l}\left(a_{1}, b_{1}\right)$ was introduced and studied by El-Ashwah and Aouf [12].

In this paper we will derive several subordination results, superordination results and sandwich results involving the operator $D_{\lambda, l}^{n}(f * g)$ and some of its special chooses of $n, l, \lambda$ and the function $g$.

\section{Definitions and Preliminaries}

In order to prove our subordinations and superordinations, we need the following definition and lemmas. 
Definition 1 ([17]). Denote by $Q$, the set of all functions $f$ that are analytic and injective on $U \backslash E(f)$, where

$$
E(f)=\left\{\zeta \in \partial U: \lim _{z \rightarrow \zeta} f(z)=\infty\right\}
$$

and are such that $f^{\prime}(\zeta) \neq 0$ for $\zeta \in \partial U \backslash E(f)$.

Lemma 1 ([24]). Let $q$ be univalent in $U$ with $q(0)=1$. Let $\alpha \in \mathbb{C}, \gamma \in \mathbb{C}^{*}$ and further assume that

$$
\operatorname{Re}\left\{1+\frac{z q^{\prime \prime}(z)}{q^{\prime}(z)}\right\}>\max \left\{0,-\operatorname{Re}\left(\frac{\alpha}{\gamma}\right)\right\} .
$$

If $p$ is analytic in $U$, and

$$
\alpha p(z)+\gamma z p^{\prime}(z) \prec \alpha q(z)+\gamma z q^{\prime}(z)
$$

then $p \prec q$ and $q$ is the best dominant.

Lemma 2 ([24]). Let $q$ be convex univalent in $U, q(0)=1$. Let $\alpha \in \mathbb{C}$, $\gamma \in \mathbb{C}^{*}$ with $\operatorname{Re}\left(\frac{\alpha}{\gamma}\right)>0$. If $p \in H[q(0), 1] \cap Q, \alpha p(z)+\gamma z p^{\prime}(z)$ is univalent in $U$ and

$$
\alpha q(z)+\gamma z q^{\prime}(z) \prec \alpha p(z)+\gamma z p^{\prime}(z)
$$

then $q \prec p$ and $q$ is the best subordinant.

\section{Sandwich Results}

Unless otherwise mentioned, we assume throughout this paper that $l \geq 0, \lambda>$ $0, n \in \mathbb{N}_{0}$ and $g$ is given by (5).

Theorem 1. Let $q(z)$ be univalent in $U$ with $q(0)=1$, and $\gamma \in \mathbb{C}^{*}$. Further, assume that

$$
\operatorname{Re}\left\{1+\frac{z q^{\prime \prime}(z)}{q^{\prime}(z)}\right\}>\max \left\{0,-\operatorname{Re}\left(\frac{1}{\gamma}\right)\right\} .
$$

If $f, g \in \mathcal{A}$ satisfy the following subordination condition

$$
\begin{aligned}
& \frac{D_{\lambda, l}^{n}(f * g)(z)}{D_{\lambda, l}^{n+1}(f * g)(z)}+\frac{\gamma(l+1)}{\lambda}\left\{1-\frac{D_{\lambda, l}^{n}(f * g)(z) D_{\lambda, l}^{n+2}(f * g)(z)}{\left[D_{\lambda, l}^{n+1}(f * g)(z)\right]^{2}}\right\} \\
\prec & q(z)+\gamma z q^{\prime}(z),
\end{aligned}
$$


then

$$
\frac{D_{\lambda, l}^{n}(f * g)(z)}{D_{\lambda, l}^{n+1}(f * g)(z)} \prec q(z)
$$

and $q$ is the best dominant.

Proof. Define the function $p$ by

$$
p(z)=\frac{D_{\lambda, l}^{n}(f * g)(z)}{D_{\lambda, l}^{n+1}(f * g)(z)}, \quad z \in U .
$$

Then the function $p$ is analytic in $U$ and $p(0)=1$. Therefore, differentiating (15) logarithmically with respect to $z$ and using the identity (8) in the resulting equation, we have

$\frac{D_{\lambda, l}^{n}(f * g)(z)}{D_{\lambda, l}^{n+1}(f * g)(z)}+\frac{\gamma(l+1)}{\lambda}\left\{1-\frac{D_{\lambda, l}^{n}(f * g)(z) D_{\lambda, l}^{n+2}(f * g)(z)}{\left[D_{\lambda, l}^{n+1}(f * g)(z)\right]^{2}}\right\}=p(z)+\gamma z p^{\prime}(z)$,

that is,

$$
p(z)+\gamma z p^{\prime}(z) \prec q(z)+\gamma z q^{\prime}(z),
$$

and Theorem 1 now follows by applying Lemma 1.

Putting $q(z)=\frac{1+A z}{1+B z}(-1 \leq B<A \leq 1)$ in Theorem 1, we obtain the following corollary:

Corollary 1. Let $\gamma \in \mathbb{C}^{*}$ such that

$$
\operatorname{Re}\left\{\frac{1-B z}{1+B z}\right\}>\max \left\{0,-\operatorname{Re}\left(\frac{1}{\gamma}\right)\right\} .
$$

If $f, g \in \mathcal{A}$ satisfy the following subordination condition

$$
\begin{aligned}
& \frac{D_{\lambda, l}^{n}(f * g)(z)}{D_{\lambda, l}^{n+1}(f * g)(z)}+\frac{\gamma(l+1)}{\lambda}\left\{1-\frac{D_{\lambda, l}^{n}(f * g)(z) D_{\lambda, l}^{n+2}(f * g)(z)}{\left[D_{\lambda, l}^{n+1}(f * g)(z)\right]^{2}}\right\} \\
\prec & \frac{1+A z}{1+B z}+\gamma \frac{(A-B) z}{(1+B z)^{2}},
\end{aligned}
$$

then

$$
\frac{D_{\lambda, l}^{n}(f * g)(z)}{D_{\lambda, l}^{n+1}(f * g)(z)} \prec \frac{1+A z}{1+B z}
$$

and the function $\frac{1+A z}{1+B z}$ is the best dominant. 
Taking $g(z)=\frac{z}{1-z}$ in Theorem 1, we obtain the following subordination result for the generalized multiplier transformation $I(n, \lambda, l)$ :

Corollary 2. Let $q$ be univalent in $U$ with $q(0)=1$, and $\gamma \in \mathbb{C}^{*}$. Further, assume that (13) holds. If $f \in \mathcal{A}$ satisfies the following subordination condition

$$
\begin{aligned}
& \frac{I(n, \lambda, l) f(z)}{I(n+1, \lambda, l) f(z)}+\frac{\gamma(l+1)}{\lambda}\left\{1-\frac{I(n, \lambda, l) f(z) I(n+2, \lambda, l) f(z)}{[I(n+1, \lambda, l) f(z)]^{2}}\right\} \\
\prec & q(z)+\gamma z q^{\prime}(z),
\end{aligned}
$$

then

$$
\frac{I(n, \lambda, l) f(z)}{I(n+1, \lambda, l) f(z)} \prec q(z)
$$

and $q$ is the best dominant.

Taking $g$ of the form (9) in Theorem 1, we obtain the following subordination result for the operator $I_{q, s, \lambda}^{n, l}\left(a_{1} ; b_{1}\right)$ :

Corollary 3. Let $q$ be univalent in $U$ with $q(0)=1$, and $\gamma \in \mathbb{C}^{*}$. Further, assume that (13) holds. If $f \in \mathcal{A}$ satisfies the following subordination condition

$$
\begin{aligned}
& \frac{I_{q, s, \lambda}^{n, l}\left(a_{1}, b_{1}\right) f(z)}{I_{q, s, \lambda}^{n+1, l}\left(a_{1}, b_{1}\right) f(z)}+\frac{\gamma(l+1)}{\lambda}\left\{1-\frac{I_{q, s, \lambda}^{n, l}\left(a_{1}, b_{1}\right) f(z) I_{q, s, \lambda}^{n+2, l}\left(a_{1}, b_{1}\right) f(z)}{\left[I_{q, s, \lambda}^{n+1, l}\left(a_{1}, b_{1}\right) f(z)\right]^{2}}\right\} \\
\prec & q(z)+\gamma z q^{\prime}(z),
\end{aligned}
$$

then

$$
\frac{I_{q, s, \lambda}^{n, l}\left(a_{1}, b_{1}\right) f(z)}{I_{q, s, \lambda}^{n+1, l}\left(a_{1}, b_{1}\right) f(z)} \prec q(z)
$$

and $q$ is the best dominant.

Taking $l=0, \lambda=1$ and $g(z)=\frac{z}{1-z}$ in Theorem 1, we obtain the following subordination result for Sălăgean operator which improves the result of Shanmugam et al. [24, Theorem 5.1] and was obtained by Nechita [18, Corollary 7]:

Corollary 4 ( $[18$, Corollary 7$])$. Let $q$ be univalent in $U$ with $q(0)=1$, and $\gamma \in \mathbb{C}^{*}$. Further, assume that (13) holds. If $f \in \mathcal{A}$ satisfies the following subordination condition:

$$
\frac{D^{n} f(z)}{D^{n+1} f(z)}+\gamma\left\{1-\frac{D^{n} f(z) D^{n+2} f(z)}{\left[D^{n+1} f(z)\right]^{2}}\right\} \prec q(z)+\gamma z q^{\prime}(z)
$$


then

$$
\frac{D^{n} f(z)}{D^{n+1} f(z)} \prec q(z)
$$

and $q$ is the best dominant.

By appealing to Lemma 2 it can be easily prove the following theorem:

Theorem 2. Let $q$ be convex univalent in $U$ with $q(0)=1$ and $\gamma \in \mathbb{C}$ with $\operatorname{Re}(\bar{\gamma})>0$. If $f, g \in \mathcal{A}$ such that $\frac{D_{\lambda, l}^{n}(f * g)(z)}{D_{\lambda, l}^{n+1}(f * g)(z)} \in H[q(0), 1] \cap Q$,

$$
\frac{D_{\lambda, l}^{n}(f * g)(z)}{D_{\lambda, l}^{n+1}(f * g)(z)}+\frac{\gamma(l+1)}{\lambda}\left\{1-\frac{D_{\lambda, l}^{n}(f * g)(z) \cdot D_{\lambda, l}^{n+2}(f * g)(z)}{\left[D_{\lambda, l}^{n+1}(f * g)(z)\right]^{2}}\right\}
$$

is univalent in $U$, and the following superordination condition

$$
\begin{aligned}
& q(z)+\gamma z q^{\prime}(z) \\
\prec & \frac{D_{\lambda, l}^{n}(f * g)(z)}{D_{\lambda, l}^{n+1}(f * g)(z)}+\frac{\gamma(l+1)}{\lambda}\left\{1-\frac{D_{\lambda, l}^{n}(f * g)(z) \cdot D_{\lambda, l}^{n+2}(f * g)(z)}{\left[D_{\lambda, l}^{n+1}(f * g)(z)\right]^{2}}\right\}
\end{aligned}
$$

holds, then

$$
q(z) \prec \frac{D_{\lambda, l}^{n}(f * g)(z)}{D_{\lambda, l}^{n+1}(f * g)(z)}
$$

and $q$ is the best subordinant.

Taking $q(z)=\frac{1+A z}{1+B z}(-1 \leq B<A \leq 1)$ in Theorem 2, we obtain the following corollary:

Corollary 5. Let $\gamma \in \mathbb{C}$ with $\operatorname{Re}(\bar{\gamma})>0$. If $f, g \in \mathcal{A}$ such that $\frac{D_{\lambda, l}^{n}(f * g)(z)}{D_{\lambda, l}^{n+1}(f * g)(z)} \in$ $H[q(0), 1] \cap Q$,

$$
\frac{D_{\lambda, l}^{n}(f * g)(z)}{D_{\lambda, l}^{n+1}(f * g)(z)}+\frac{\gamma(l+1)}{\lambda}\left\{1-\frac{D_{\lambda, l}^{n}(f * g)(z) D_{\lambda, l}^{n+2}(f * g)(z)}{\left[D_{\lambda, l}^{n+1}(f * g)(z)\right]^{2}}\right\}
$$

is univalent in $U$, and the following superordination condition

$$
\begin{aligned}
& \frac{1+A z}{1+B z}+\gamma \frac{(A-B) z}{(1+B z)^{2}} \\
\prec & \frac{D_{\lambda, l}^{n}(f * g)(z)}{D_{\lambda, l}^{n+1}(f * g)(z)}+\frac{\gamma(l+1)}{\lambda}\left\{1-\frac{D_{\lambda, l}^{n}(f * g)(z) D_{\lambda, l}^{n+2}(f * g)(z)}{\left[D_{\lambda, l}^{n+1}(f * g)(z)\right]^{2}}\right\}
\end{aligned}
$$


holds, then

$$
\frac{1+A z}{1+B z} \prec \frac{D_{\lambda, l}^{n}(f * g)(z)}{D_{\lambda, l}^{n+1}(f * g)(z)}
$$

and $q$ is the best subordinant.

Taking $g(z)=\frac{z}{1-z}$ in Theorem 2, we obtain the following superordination result for the generalized multiplier transformation $I(n, \lambda, l)$ :

Corollary 6. Let $q$ be convex univalent in $U$ with $q(0)=1$, and let $\gamma \in \mathbb{C}$ with $\operatorname{Re}(\bar{\gamma})>0$. If $f, g \in \mathcal{A}$ such that $\frac{I(n, \lambda, l) f(z)}{I(n+1, \lambda, l) f(z)} \in H[q(0), 1] \cap Q$,

$$
\frac{I(n, \lambda, l) f(z)}{I(n+1, \lambda, l) f(z)}+\frac{\gamma(l+1)}{\lambda}\left\{1-\frac{I(n, \lambda, l) f(z) \cdot I(n+2, \lambda, l) f(z)}{[I(n+1, \lambda, l) f(z)]^{2}}\right\}
$$

is univalent in $U$, and the following superordination condition

$$
\begin{aligned}
& q(z)+\gamma z q^{\prime}(z) \\
\prec & \frac{I(n, \lambda, l) f(z)}{I(n+1, \lambda, l) f(z)}+\frac{\gamma(l+1)}{\lambda}\left\{1-\frac{I(n, \lambda, l) f(z) \cdot I(n+2, \lambda, l) f(z)}{[I(n+1, \lambda, l) f(z)]^{2}}\right\}
\end{aligned}
$$

holds, then

$$
q(z) \prec \frac{I(n, \lambda, l) f(z)}{I(n+1, \lambda, l) f(z)}
$$

and $q$ is the best subordinant.

Taking $g$ of the form (9) in Theorem 2, we obtain the following superordination result for the operator $I_{q, s, \lambda}^{n, l}\left(a_{1} ; b_{1}\right)$ :

Corollary 7. Let $q$ be convex univalent in $U$ with $q(0)=1$, and let $\gamma \in \mathbb{C}$ with $\operatorname{Re}(\bar{\gamma})>0$. If $f, g \in \mathcal{A}$ such that $\frac{I_{q, s, \lambda}^{n, l}\left(a_{1}, b_{1}\right) f(z)}{I_{q, s, \lambda}^{n+1, l}\left(a_{1}, b_{1}\right) f(z)} \in H[q(0), 1] \cap Q$,

$$
\frac{I_{q, s, \lambda}^{n, l}\left(a_{1}, b_{1}\right) f(z)}{I_{q, s, \lambda}^{n+1, l}\left(a_{1}, b_{1}\right) f(z)}+\frac{\gamma(l+1)}{\lambda}\left\{1-\frac{I_{q, s, \lambda}^{n, l}\left(a_{1}, b_{1}\right) f(z) I_{q, s, \lambda}^{n+2, l}\left(a_{1}, b_{1}\right) f(z)}{\left[I_{q, s, \lambda}^{n+1, l}\left(a_{1}, b_{1}\right) f(z)\right]^{2}}\right\}
$$

is univalent in $U$, and the following superordination condition

$$
\begin{aligned}
& q(z)+\gamma z q^{\prime}(z) \\
\prec & \frac{I_{q, s, \lambda}^{n, l}\left(a_{1}, b_{1}\right) f(z)}{I_{q, s, \lambda}^{n+1, l}\left(a_{1}, b_{1}\right) f(z)}+\frac{\gamma(l+1)}{\lambda}\left\{1-\frac{I_{q, s, \lambda}^{n, l}\left(a_{1}, b_{1}\right) f(z) I_{q, s, \lambda}^{n+2, l}\left(a_{1}, b_{1}\right) f(z)}{\left[I_{q, s, \lambda}^{n+1, l}\left(a_{1}, b_{1}\right) f(z)\right]^{2}}\right\}
\end{aligned}
$$


holds, then

$$
q(z) \prec \frac{I_{q, s, \lambda}^{n, l}\left(a_{1}, b_{1}\right) f(z)}{I_{q, s, \lambda}^{n+1, l}\left(a_{1}, b_{1}\right) f(z)}
$$

and $q$ is the best subordinant.

Taking $l=0, \lambda=1$ and $g(z)=\frac{z}{1-z}$ in Theorem 2, we obtain the following superordination result for Sălăgean operator which improves the result of Shanmugam et al.[24, Theorem 5.2] and was obtained by Nechita [18, Corollary 12]:

Corollary 8 ([18, Corollary 12]). Let $q$ be convex univalent in $U$ with $q(0)=$ 1 , and let $\gamma \in \mathbb{C}$ with $\operatorname{Re}(\bar{\gamma})>0$. If $f \in \mathcal{A}$ such that $\frac{D^{n} f(z)}{D^{n+1} f(z)} \in H[q(0), 1] \cap Q$,

$$
\frac{D^{n} f(z)}{D^{n+1} f(z)}+\gamma\left\{1-\frac{D^{n} f(z) \cdot D^{n+2} f(z)}{\left[D^{n+1} f(z)\right]^{2}}\right\}
$$

is univalent in $U$, and the following superordination condition

$$
q(z)+\gamma z q^{\prime}(z) \prec \frac{D^{n} f(z)}{D^{n+1} f(z)}+\gamma\left\{1-\frac{D^{n} f(z) \cdot D^{n+2} f(z)}{\left[D^{n+1} f(z)\right]^{2}}\right\}
$$

holds, then

$$
q(z) \prec \frac{D^{n} f(z)}{D^{n+1} f(z)}
$$

and $q$ is the best subordinant.

Combining Theorem 1 and Theorem 2 we get the following sandwich theorem for the linear operator $D_{\lambda, l}^{n}$ :

Theorem 3. Let $q_{1}$ be convex univalent in $U$ with $q_{1}(0)=1, \gamma \in \mathbb{C}$ with $\operatorname{Re}(\bar{\gamma})>0, q_{2}(z)$ be univalent in $U$ with $q_{2}(0)=1$, and satisfies 13 . If $f, g \in \mathcal{A}$ such that $\frac{D_{\lambda, l}^{n}(f * g)(z)}{D_{\lambda, l}^{n+1}(f * g)(z)} \in H\left[q_{2}(0), 1\right] \cap Q$,

$$
\frac{D_{\lambda, l}^{n}(f * g)(z)}{D_{\lambda, l}^{n+1}(f * g)(z)}+\frac{\gamma(l+1)}{\lambda}\left\{1-\frac{D_{\lambda, l}^{n}(f * g)(z) \cdot D_{\lambda, l}^{n+2}(f * g)(z)}{\left[D_{\lambda, l}^{n+1}(f * g)(z)\right]^{2}}\right\}
$$

is univalent in $U$, and

$$
\begin{aligned}
& q_{1}(z)+\gamma z q_{1}^{\prime}(z) \\
\prec & \frac{D_{\lambda, l}^{n}(f * g)(z)}{D_{\lambda, l}^{n+1}(f * g)(z)}+\frac{\gamma(l+1)}{\lambda}\left\{1-\frac{D_{\lambda, l}^{n}(f * g)(z) \cdot D_{\lambda, l}^{n+2}(f * g)(z)}{\left[D_{\lambda, l}^{n+1}(f * g)(z)\right]^{2}}\right\} \\
\prec & q_{2}(z)+\gamma z q_{2}^{\prime}(z)
\end{aligned}
$$


holds, then

$$
q_{1}(z) \prec \frac{D_{\lambda, l}^{n}(f * g)(z)}{D_{\lambda, l}^{n+1}(f * g)(z)} \prec q_{2}(z)
$$

and $q_{1}$ and $q_{2}$ are, respectively, the best subordinant and the best dominant.

Taking $q_{i}(z)=\frac{1+A_{i} z}{1+B_{i} z}\left(i=1,2 ;-1 \leq B_{2} \leq B_{1}<A_{1} \leq A_{2} \leq 1\right)$ in Theorem 3 we obtain the following corollary:

Corollary 9. Let $\gamma \in \mathbb{C}$ with $\operatorname{Re}(\bar{\gamma})>0$. If $f, g \in \mathcal{A}$ such that $\frac{D_{\lambda, l}^{n}(f * g)(z)}{D_{\lambda, l}^{n+1}(f * g)(z)} \in$ $H[q(0), 1] \cap Q$,

$$
\frac{D_{\lambda, l}^{n}(f * g)(z)}{D_{\lambda, l}^{n+1}(f * g)(z)}+\frac{\gamma(l+1)}{\lambda}\left\{1-\frac{D_{\lambda, l}^{n}(f * g)(z) \cdot D_{\lambda, l}^{n+2}(f * g)(z)}{\left[D_{\lambda, l}^{n+1}(f * g)(z)\right]^{2}}\right\}
$$

is univalent in $U$, and

$$
\begin{aligned}
& \frac{1+A_{1} z}{1+B_{1} z}+\gamma \frac{\left(A_{1}-B_{1}\right) z}{\left(1+B_{1} z\right)^{2}} \\
\prec & \frac{D_{\lambda, l}^{n}(f * g)(z)}{D_{\lambda, l}^{n+1}(f * g)(z)}+\frac{\gamma(l+1)}{\lambda}\left\{1-\frac{D_{\lambda, l}^{n}(f * g)(z) \cdot D_{\lambda, l}^{n+2}(f * g)(z)}{\left[D_{\lambda, l}^{n+1}(f * g)(z)\right]^{2}}\right\} \\
\prec & \frac{1+A_{2} z}{1+B_{2} z}+\gamma \frac{\left(A_{2}-B_{2}\right) z}{\left(1+B_{2} z\right)^{2}}
\end{aligned}
$$

holds, then

$$
\frac{1+A_{1} z}{1+B_{1} z} \prec \frac{D_{\lambda, l}^{n}(f * g)(z)}{D_{\lambda, l}^{n+1}(f * g)(z)} \prec \frac{1+A_{2} z}{1+B_{2} z}
$$

and $\frac{1+A_{1} z}{1+B_{1} z}$ and $\frac{1+A_{2} z}{1+B_{2} z}$ are, respectively, the best subordinant and the best dominant.

Taking $l=0, \lambda=1$ and $g(z)=\frac{z}{1-z}$ in Theorem 3 , we obtain the following sandwich result for Sălăgean operator which improves the result of Shanmugam et al. [24, Theorem 5.3]:

Corollary 10. Let $q_{1}$ be convex univalent in $U$ with $q_{1}(0)=1, \gamma \in \mathbb{C}$ with $\operatorname{Re}(\bar{\gamma})>0, q_{2}$ be univalent in $U$ with $q_{2}(0)=1$, and satisfies (13). If $f \in \mathcal{A}$ such that $\frac{D^{n} f(z)}{D^{n+1} f(z)} \in H\left[q_{2}(0), 1\right] \cap Q$,

$$
\frac{D^{n} f(z)}{D^{n+1} f(z)}+\gamma\left\{1-\frac{D^{n} f(z) \cdot D^{n+2} f(z)}{\left[D^{n+1} f(z)\right]^{2}}\right\}
$$


is univalent in $U$, and

$q_{1}(z)+\gamma z q_{1}^{\prime}(z) \prec \frac{D^{n} f(z)}{D^{n+1} f(z)}+\gamma\left\{1-\frac{D^{n} f(z) \cdot D^{n+2} f(z)}{\left[D^{n+1} f(z)\right]^{2}}\right\} \prec q_{2}(z)+\gamma z q_{2}^{\prime}(z)$

holds, then

$$
q_{1}(z) \prec \frac{D^{n} f(z)}{D^{n+1} f(z)} \prec q_{2}(z)
$$

and $q_{1}$ and $q_{2}$ are, respectively, the best subordinant and the best dominant.

Remark 1. Combining Corollary 2 and Corollary 5, we obtain a similar sandwich theorem for the generalized multiplier transformation $I(n, \lambda, l)$;

Remark 2. Combining Corollary 3 and Corollary 6, we obtain a similar sandwich theorems for the operator $I_{q, s, \lambda}^{n, l}\left(a_{1}, b_{1}\right)$;

Remark 3. Taking $l=0$ and $g(z)=\frac{z}{1-z}$ in Theorems 1, 2 and 3, respectively, we obtain the results of Nechita [18, Theorems 5, 10 and Corollary 13, respectively];

Remark 4. Taking $n=l=0, \lambda=1$ and $g(z)=\frac{z}{1-z}$ in Theorems 1,2 and 3 , respectively, we obtain the results of Shanmugam et al.[24, Theorems 3.1, 3.2 and Corollary 3.3, respectively].

\section{Acknowledgement}

The author thanks the referees for their valuable suggestions which led to improvement of the paper.

\section{References}

[1] R.M. Ali, V. Ravichandran, and K.G Subramanian, Differential sandwich theorems for certain analytic functions, Far East J. Math. Sci., 15, (2004), 87-94.

[2] F.M. Al-Oboudi, On univalent functions defined by a generalized Sălăgean operator, Internat. J. Math. Math. Sci., 27, (2004), 1429-1436.

[3] M.K. Aouf and A. O. A. O. Mostafa, Sandwich theorems for analytic functions defined by convolution, Acta Univ. Apulensis, 21, (2010), 7-20.

[4] S. D. Bernardi, Convex and starlike univalent functions, Trans. Amer. Math. Soc., 135, (1969), 429-446. 
[5] T. Bulboacă, Classes of first order differential superordinations, Demonstratio Math., 35, (2002), 287-292.

[6] T. Bulboacă, Differential Subordinations and Superordinations, Recent Results, House of Scientific Book Publ., Cluj-Napoca, 2005.

[7] B. C. Carlson and D. B. Shaffer, Starlike and prestarlike hypergeometric functions, SIAM J. Math. Anal., 15, (1984), 737-745.

[8] A. Cătaş, G. I. Oros, and G Oros, Differential subordinations associated with multiplier transformations, Abstract Appl. Anal., 2008,ID 845724, (2008), 1-11.

[9] N. E. Cho and T. G. Kim, Multiplier transformations and strongly close-to-convex functions, Bull. Korean Math. Soc., 40, (2003), 399-410.

[10] J. Dziok and H. M. Srivastava, Classes of analytic functions associated with the generalized hypergeometric function, Appl. Math. Comput., 103, (1999), 1-13.

[11] J. Dziok and H. M. Srivastava, Certain subclasses of analytic functions associated with the generalized hypergeometric function, Integral Transform. Spec. Funct., 14, (2003), 7-18.

[12] R. M. El-Ashwah and M. K. Aouf, Differential subordination and superordination for certain subclasses of $p$-valent functions, Math. Comput. Modelling, 51, (2010), 349-360.

[13] Yu. E. Hohlov, Operators and operations in the univalent functions, Izv. Vysshh. Učebn. Zaved. Mat., 10, (1978), 83-89.

[14] R. J. Libera, Some classes of regular univalent functions, Proc. Amer. Math. Soc., 16, (1965), 755-758.

[15] A. E. Livingston, On the radius of univalence of certain analytic functions, Proc. Amer. Math. Soc., 17, (1966), 352-357.

[16] S. S. Miller and P. T. Mocanu, Differential Subordination: Theory and Applications, Series on Monographs and Textbooks in Pure and Applied Mathematics, Vol. 225, Marcel Dekker Inc., New York and Basel, 2000.

[17] S. S. Miller and P. T. Mocanu, Subordinates of differential superordinations, Complex Variables, 48, (2003), 815-826.

[18] V. O. Nechita, Differential subordinations and superordinations for analytic functions defined by the generalized Sălăgean derivative, Acta Univ. Apulensis, 16, (2008), 143-156.

[19] S. Owa and H. M. Srivastava, Univalent and starlike generalized hypergeometric functions, Canad. J. Math., 39, (1987), 1057-1977.

[20] St. Ruscheweyh, New criteria for univalent functions, Proc. Amer. Math. Soc., 49, (1975), 109-115.

[21] H. Saitoh, A linear operator and its applications of fiest order differential subordinations, Math. Japon., 44, (1996), 31-38.

[22] G. S. Sălăgean, Subclasses of univalent functions, Lecture Notes in Math. (SpringerVerlag), 1013, (1983), 362-372. 
[23] C. Selvaraj and K. R. Karthikeyan, Differential subordination and superordination for certain subclasses of analytic functions, Far East J. Math. Sci., 29, (2008), 419-430.

[24] T. N. Shanmugam, V. Ravichandran, and S. Sivasubramanian, Differential sandwich theorems for some subclasses of analytic functions, J. Australian Math. Anal. Appl., 3, Art. 8, (2006), 1-11.

[25] N. N. Tuneski, On certain sufficient conditions for starlikeness, Internat. J. Math. Math. Sci., 23, (2000), 521-527.

Tamer Mohamed Seoudy

Department of Mathematics

Faculty of Science

Fayoum University

Fayoum 63514, Egypt

E-mail: tms00@fayoum.edu.eg

Submitted: 15.11.2012

Accepted: 20.03.2013 